\title{
35: Introduction to Case Studies
}

The foregoing chapters of this book have been concerned with the instruments used in financing international trade, assessment of the risks involved and the documentation used to evidence trade and gain payment. Part IV illustrates how financing techniques can be adapted and applied in the context of real deals.

All of the case studies in Part IV really happened. Duties of confidentiality and discretion owed to former employers, clients and colleagues require that the details of transactions and particularly the names of the counterparties be disguised. These case studies should adequately fulfill their purpose nevertheless. 